\title{
RELATED REPRESENTATION THEOREMS FOR RINGS, SEMIRINGS, NEAR-RINGS AND SEMI-NEAR-RINGS BY PARTIAL TRANSFORMATIONS AND PARTIAL ENDOMORPHISMS
}

\author{
by HANNS JOACHIM WEINERT
}

(Received 21st October 1975)

\section{Introduction}

Fundamental statements for (associative) rings are that (a) the endomorphisms of each commutative group $(U,+)$ form a ring and (b) each ring may be embedded in such a ring of endomorphisms. In order to generalise these theorems to groups and rings whose addition may not be commutative, one has to deal with partial endomorphisms. But these ring-theoretical Theorems $4 \mathrm{a}$ and $4 \mathrm{~b}$ turn out to be specialisations of similar ones for semi-near-rings, near-rings and semirings, developed here in Section 2 after some preliminaries on semi-near-rings in Section 1. A glance at Definition 1 and the ring-theoretical theorems and remarks at the end of Section 2 may give more orientation.

As shown by references given in the text, parts of our statements concerning semi-near-rings and near-rings are known, at least in more specialised versions. Our emphasis here is with the strong and useful analogy and relationship. Only for some semirings this analogy is not as complete as one could wish; we shall discuss this situation dealing with semirings in Section 2 and give corresponding examples in Section 3. In this context we obtain some results concerning conditions under which the set $E(S)$ of all endomorphisms of a semigroup $(S,+)$ is embeddable in a semiring. This problem, raised by A. H. Clifford and Mrs. M. P. Grillet, has two different interpretations (see Section 3, Definition 2), leading to different answers.

\section{Preliminaries}

An algebra $(S,+, \cdot)$ with two binary operations is a (left-distributive) semi-near-ring if $(S,+)$ and $(S, \cdot)$ are semigroups and $a(b+c)=$ $a b+a c$ holds for all $a, b, c \in S$. This concept (restricted to semi-near-rings with an annihilating zero) was introduced in (7); see also (6) and (5). Throughout this paper, neutral elements with respect to an addition or a multiplication are simply called zero or identity, respectively. If $(S,+, \cdot)$ has a zero $o$, an element $n \in S$ is called a left-annihilator of $(S,+, \cdot)$ if $n a=o$ 
holds for all $a \in S$; the zero $o$ itself need not be a left-annihilator or a right-annihilator, but the latter follows from left-distributivity if $(S,+)$ is (left or right) cancellative. In each semi-near-ring, the set $S^{2}$ defined by

$$
S^{2}=\left\{\sum_{i=1}^{n} a_{i} b_{i} \mid a_{i}, b_{i} \in S, n=1,2, \ldots\right\} \subseteq S
$$

forms a subsemigroup $\left(S^{2},+\right)$ of $(S,+)$. Further, we need slight generalisations of concepts due to (1) and call an element $d$ of a semi-near-ring $(S,+, \cdot)$ distributive if $(a+b) d=a d+b d$ holds for all $a, b \in S$. If the subset $D \subseteq S$ of all distributive elements of $S$ is not empty, $(D, \cdot)$ clearly is a subsemigroup of $(S, \cdot)$. Now let $\left(D^{\prime}, \cdot\right)$ be a subsemigroup of $(D, \cdot)$, in short a distributive subsemigroup of $(S,+, \cdot)$; then

$$
\left\langle D^{\prime}\right\rangle=\left\{\sum_{i=1}^{n} d_{i} \mid d_{i} \in D^{\prime}, n=1,2, \ldots\right\} \subseteq S
$$

forms a subsemi-near-ring of $(S,+, \cdot)$, and we call such a subsemi-near-ring distributively generated.

Finally, a semi-near-ring $(S,+, \cdot)$ is called a semiring or a near-ring, if all elements of $S$ are distributive or if $(S,+)$ is a group, respectively. If both hold, we have the concept of an additively not necessarily commutative ring (distributive near-ring), simply called ring in this paper. Results and examples concerning such generalised rings as well as more references are given in (8).

\section{Partial transformations and endomorphisms of semigroups}

Definition 1. Let $(G,+) \supseteq(U,+) \supseteq(W,+)$ be a chain of semigroups [of groups]. Then a mapping $\varphi: G \rightarrow U \rightarrow W$, i.e.

$$
g^{\varphi} \in U \text { for all } g \in G, \quad u^{\varphi} \in W \text { for all } u \in U
$$

is called a partial transformation of the semigroups $(G, U, W,+)$ [of the groups $(G, U, W,+)]$. In particular, if also

$$
(u+v)^{\varphi}=u^{\varphi}+v^{\varphi} \quad \text { for all } u, v \in U
$$

is satisfied, we call $\varphi$ a partial endomorphism of $(G, U, W,+)$.

Remark. Throughout this paper, addition will only be needed for elements from $U$. Hence we replace $(G,+)$ by a set $G$ containing $(U,+)$, using further the expressions "of the semigroups $(G, U, W,+)$ " or "of the groups $(G, U, W,+)$ ".

Theorem 1a. The set $T=T(G, U, W,+)$ of all partial transformation $\varphi, \psi, \ldots$ of the semigroups $(G, U, W,+)$ endowed with the usual operations

$$
x^{\varphi+\psi}=x^{\varphi}+x^{\psi} \text { and } x^{\varphi \cdot \psi}=\left(x^{\varphi}\right)^{\psi} \quad \text { for all } x \in G
$$


is a semi-near-ring $(T,+, \cdot){ }^{1}$ The subset $E=E(G, U, W,+) \subseteq T$ of partial endomorphisms is either empty or a distributive subsemigroup $(E, \cdot)$ of $(T,+, \cdot)$. If $G \supset U$, then $E$ coincides with the set $D_{T}$ of all distributive elements of $(T,+, \cdot)$.

Proof. For each $w \in W$ the constant mapping $\gamma_{W}$ defined by $x^{\gamma_{w}}=w$ for all $x \in G$ is an element of $T$, hence $T \neq \emptyset$. The axioms establishing $(T,+, \cdot)$ as a semi-near-ring including left distributivity follow directly from the definitions, using the facts that $(U,+)$ and $(W,+)$ are semigroups.

Further, for each idempotent element $w+w=w$ of $(W,+)$, the constant mapping $\gamma_{w}$ is even a partial endomorphism of the semigroups $(G, U, W,+)$. But $E$ may be empty if $(W,+)$ has no idempotent, for instance, if $(U,+)$ has one. If $E \neq \emptyset$, clearly $(E, \cdot)$ is a semigroup, and $E \subseteq D_{T}$, i.e. $(\varphi+\psi) \eta=$ $\varphi \eta+\psi \eta$ for all $\varphi, \psi \in T, \eta \in E$ follows from

$$
x^{(\varphi+\psi) \eta}=\left(x^{\varphi}+x^{\psi}\right)^{\eta}=\left(x^{\varphi}\right)^{\eta}+\left(x^{\psi}\right)^{\eta}=x^{\varphi \eta+\psi \eta}
$$

for all $x \in G$, depending essentially on $\eta$ satisfying (2.2).

Now suppose $G \supset U$. Clearly $E=D_{T}$ if $E=T$. If $E \subset T$, let $\tau$ be any element out of $T \backslash E$. Then there exist $u, v \in U$ such that $(u+v)^{\tau} \neq u^{\tau}+v^{\tau}$ and clearly partial transformations (even partial endomorphisms if $E \neq \emptyset$ ) $\varphi, \psi \in T$ such that $x^{\varphi}=u$ and $x^{\psi}=v$ for some $x \in G \backslash U$. But then for this $x$ one has

$$
x^{(\varphi+\psi) \tau}=(u+v)^{\tau} \neq u^{\tau}+v^{\tau}=x^{\varphi \tau+\psi \tau},
$$

showing that $\tau$ is not a distributive element of $(T,+, \cdot)$.

Clearly, commutativity of $(U,+)$ implies commutativity of $(T,+)$; we remark that the converse holds if $G \supset U$.

Theorem 1b. Each semi-near-ring $(S,+, \cdot)$ is isomorphic to a subseminear-ring of the semi-near-ring $(T,+, \cdot)$ of partial transformations with $T=T\left(G, S, S^{2},+\right)$, where $G$ is any set properly containing the semigroups $(S,+) \supseteq\left(S^{2},+\right)$. The desired monomorphism $\mu: S \rightarrow T$ is given for each $a \in S$ by

$$
a \rightarrow \mu(a)=\alpha: x \rightarrow x^{\alpha}=\left\{\begin{array}{cl}
x \cdot a & \text { if } x \in S \\
a & \text { if } x \in G \backslash S .
\end{array}\right.
$$

In particular, $\mu(d)$ is a partial endomorphism of $\left(G, S, S^{2},+\right)$ if and only if $d$ is a distributive element of $(S,+, \cdot)$.

\footnotetext{
' This statement restricted to $G=U=W$ and a corresponding version of parts of Theorem $1 \mathrm{~b}$ are given in (5).
} 
Proof. Clearly each $\mu(a)=\alpha$ is an element of $T\left(G, S, S^{2},+\right)$ and $\mu: S \rightarrow T$ is injective as $G \supset S .{ }^{2}$ For each $x \in S$ or $x \in G \backslash S$ one has

$$
\begin{aligned}
x^{\mu(a) \mu(b)} & =\left\{\begin{array}{l}
(x \cdot a)^{\mu(b)}=x \cdot a \cdot b \\
a^{\mu(b)}=a \cdot b
\end{array}\right\}=x^{\mu(a \cdot b)} \\
x^{\mu(a)+\mu(b)}=x^{\mu(a)}+x^{\mu(b)} & =\left\{\begin{array}{c}
x a+x b=x(a+b) \\
a+b
\end{array}\right\}=x^{\mu(a+b)} .
\end{aligned}
$$

Finally, $\mu(d)$ satisfies (2.2) with $U=S$ iff $d$ is distributive since

$$
(x+y)^{\mu(d)}=(x+y) d=x d+y d=x^{\mu(d)}+y^{\mu(d)} .
$$

In particular if, in Theorem 1a, the semigroups $(U,+) \supseteq(W,+)$ have a common zero $o$, the constant mapping $\gamma_{o}=\omega$ (already known to satisfy $\left.\omega \in E \subseteq D_{T} \subseteq T\right)$ is the zero of $(T,+, \cdot)$ and a right annihilator. Moreover, if $(U,+) \supseteq(W,+)$ are groups, each $\varphi \in T$ defines by $x^{-\varphi}=-\left(x^{\varphi}\right)$ again a partial transformation $-\varphi \in T$, and clearly $\varphi+(-\varphi)=\omega$. Conversely, each near-ring $(S,+, \cdot)$ has a right annihilating zero $o$. Therefore, the following theorems essentially known in near-ring theory (for similar results and some applications see (3), (4)) are simply obtained as specialisations of our theorems above:

Theorem 2a. The set $T=T(G, U, W,+)$ of all partial transformations of the groups $(G, U, W,+)$ is a near-ring $(T,+, \cdot)$. The subset $E=$ $E(G, U, W,+)$ of partial endomorphisms is a distributive subsemigroup $(E, \cdot)$ of $(T,+, \cdot)$, containing at least the zero $\gamma_{o}=\omega$ of $T$, and $E=D_{T}$ if $G \supset U$.

Theorem 2b. Each near-ring $(S,+, \cdot)$ is isomorphic by (2.4) to a subnear-ring of the near-ring $(T,+, \cdot)$ with $T=T\left(G, S, S^{2},+\right), G \supset S$; in particular one has $\mu(o)=\gamma_{o}=\omega$.

In order to get similar theorems for semirings, by the results concerning distributivity in Theorem $1 \mathrm{a}$ and in Theorem $1 \mathrm{~b}$ one has to deal with partial endomorphisms of semigroups. But such a set $E=E(G, U, W,+)$ is additively closed if and only if for all $\varphi, \psi \in E$ and all $u, v \in U$ the right hand sums

$$
\begin{aligned}
(u+v)^{\varphi+\psi} & =u^{\varphi}+v^{\varphi}+u^{\psi}+v^{\psi} \\
u^{\varphi+\psi}+v^{\varphi+\psi} & =u^{\varphi}+u^{\psi}+v^{\varphi}+v^{\psi}
\end{aligned}
$$

contained in $W$ are equal. Defining a semigroup $(W,+)$ to be $s u b$ commutative (cf. (2)) if

$$
a+b+c+d=a+c+b+d \text { for all } a, b, c, d \in W,
$$

\footnotetext{
${ }^{2}$ Choosing $G=S, \mu$ is injective iff $x a=x b$ for all $x \in S$ implies $a=b$, i.e. iff the semigroup ( $\left.S, \cdot\right)$ is left reductive.
} 
we have a sufficient condition which seems to be very natural, and we obtain from Theorem la:

Theorem 3a. The set $E=E(G, U, W,+)$ of all partial endomorphisms of the semigroups $(G, U, W,+)$ is a semiring $(E,+, \cdot)$ if $E \neq \emptyset$ and $(W,+)$ is subcommutative.

Now we start with a semiring $(S,+, \cdot)$ and look at $E=E\left(G, S, S^{2},+\right)$. But even in this case $E$ need not be additively closed, as we shall show by examples in Section 3. Hence the representation theorem for semirings obtained by Theorem $1 \mathrm{~b}$ reads as follows:

Theorem 3b. Each semiring $(S,+, \cdot)$ is isomorphic by (2.4) to a subsemiring $\mu(S)$ of the semi-near-ring $(T,+, \cdot)$ of partial transformations of semigroups with $T=T\left(G, S, S^{2},+\right), G \supset S$. Moreover, $\mu(S)$ is contained in the set $E=E\left(G, S, S^{2},+\right)$ of partial endomorphisms of the semigroups $\left(G, S, S^{2},+\right)$, itself forming a semiring if $\left(S^{2},+\right)$ is subcommutative, but not in general.

Clearly, in any case one has $\mu(S) \subseteq E \subseteq\langle E\rangle$, but also the distributively generated subsemi-near-ring $(\langle E\rangle,+, \cdot)$ of $(T,+, \cdot)$ need not be a semiring if $\left(S^{2},+\right)$ is not subcommutative (see Section 3 ). On the other hand, many semirings $(S,+, \cdot)$ have this property, and in general $\left(S^{2},+\right)$ is not so far away from subcommutativity because of

$$
a c+a d+b c+b d=(a+b)(c+d)=a c+b c+a d+b d .
$$

In particular, $\left(S^{2},+\right)$ is even commutative if it is cancellative. Therefore, applying our theorems to rings, we obtain:

Theorem 4a. The set $E=E(G, U, W,+)$ of all partial endomorphisms of groups $(G, U, W,+)$ with commutative $(W,+)$ is a ring $(E,+, \cdot)$, whose addition is commutative if the group $(U,+)$ is commutative.

Theorem 4b. Each ring $(S,+, \cdot)$ is by (2.4) isomorphic to a subring of the ring $(E,+, \cdot)$ of partial endomorphisms of groups with $E=$ $E\left(G, S, S^{2},+\right), G \supset S$.

Remark. In the additively commutative case Theorem $4 \mathrm{a}$ is usually given with $G=U=W$, and Theorem $4 \mathrm{~b}$ holds with $S=G=U$ iff the ring $(S,+, \cdot)$ is left reductive (cf. footnote 2$)$. If not, the ring can be embedded in a ring with identity before applying the theorem in this form. But a ring whose addition is not commutative has nonzero annihilators (cf. (8)), hence such a ring $(S,+, \cdot)$ is neither left reductive nor embeddable in a ring with identity. Therefore, in order to get $\mu: S \rightarrow E$ injective, $G \supset S=U$ as well as a commutative subgroup $W=S^{2}$ are indispensable in our theorems. 


\section{Embedding of sets of endomorphisms in semirings}

We shall give examples of semirings $(S,+, \cdot)$ such that $E\left(G, S, S^{2},+\right)$ with respect to the operations (2.3) is not additively closed, hence not a semiring. Thereby arises the question whether $(E,+, \cdot)$ is at least embeddable in a semiring. But this problem has two essentially different interpretations:

Definition 2. Let $E=E(G, U, W,+)$ be the set of all partial endomorphisms of the semigroups $(G, U, W,+)$, regarded as a partial subalgebra of $(T,+, \cdot)$ with $T=T(G, U, W,+)$. Then $E$ is called weakly embeddable in a semiring $(R,+, \cdot)$ if there exists a monomorphism $f$ of $(E,+, \cdot)$ into $(R,+, \cdot)$. Note that in this case only

$$
f(\varphi+\psi)=f(\varphi)+f(\psi) \text { for all } \varphi, \psi \in E \text { such that } \varphi+\psi \in E
$$

is demanded. If it is possible to extend $f$ to a monomorphism $\hat{f}$ of $(\hat{E},+, \cdot)$ into $(R,+, \cdot), E \subseteq \hat{E} \subseteq T$, such that

$$
\hat{f}(\varphi+\psi)=\hat{f}(\varphi)+\hat{f}(\psi) \text { for all } \varphi, \psi \in E
$$

holds, then $E$ is called strongly embeddable in the semiring $(R,+, \cdot)$.

The following statement is obvious:

Proposition 1. A set $E=E(G, U, W,+)$ of all partial endomorphisms of semigroups is strongly embeddable in a semiring if and only if the distributively generated semi-near-ring $(\langle E\rangle,+, \cdot)$ is a semiring. In this case, $(\langle E\rangle,+, \cdot)$ is the smallest semiring in which $E$ is strongly embeddable.

Now we can summarise the purposes of the following two examples:

(a) As claimed in Section 2, there are semirings $(S,+, \cdot)$ such that $E=E\left(S, S, S^{2},+\right)$, hence $E\left(G, S, S^{2},+\right)$ for each set $G \supseteq S$, is not additively closed and $(\langle E\rangle,+, \cdot)$ is not distributive.

(b) By the last statement and Proposition 1 in both cases $E$ is not strongly embeddable in any semiring. But in the first example $E$ will be weakly embeddable in a semiring, in the second one not.

(c) We succeed in constructing our semirings $(S,+, \cdot)$ such that $S^{2}=S$, so in addition our examples verify (b) also for the set $E=E(S, S, S,+)=$ $E(S)$ of all endomorphisms of a semigroup $(S,+)$.

Remark. An example of a semigroup $\left(S^{0},+\right)$ such that $E\left(S^{0}\right)$ is not embeddable into a semiring (more precisely: not weakly embeddable) was constructed in the second part of (2). But we do not see how to add a suitable semiring-multiplication to it and feel that our examples are simpler. $^{3}$

\footnotetext{
${ }^{3}$ There are two confusing misprints on page 349 of (2): On line 2 replace " $p \geqq 1$ " by " $p \leqq 1$ ", on line 7 replace " $\rho\left(n^{\prime}\right)=0$ if $n^{\prime} \neq 0$ " by " $\rho\left(n^{\prime}\right)=1$ if $n^{\prime}=0$ ".
} 
Example 1. The semigroup $(S,+)$ generated by two elements $a$ and $b$, subject to the relations $2 a=a, 2 b=b, a+b+a=b+a+b(=z)$ is given by the table

\begin{tabular}{c|ccccc}
+ & $a$ & $b$ & $a+b$ & $b+a$ & $z$ \\
\hline$a$ & $a$ & $a+b$ & $a+b$ & $z$ & $z$ \\
$b$ & $b+a$ & $b$ & $z$ & $b+a$ & $z$ \\
$a+b$ & $z$ & $a+b$ & $z$ & $z$ & $z$ \\
$b+a$ & $b+a$ & $z$ & $z$ & $z$ & $z$ \\
$z$ & $z$ & $z$ & $z$ & $z$ & $z$
\end{tabular}

To illustrate $(S,+)$ not being subcommutative we note

$$
a+a+b+b=a+b \neq z=a+b+a+b .
$$

It is easily seen that $(S,+, \cdot)$ becomes a semiring with $S^{2}=S$ if we define

$$
a \cdot a=a, b \cdot b=b, \quad \text { all other products to be } z \text {. }
$$

Each endomorphism $\varphi$ of $(S,+)$, i.e. each element of $E=E\left(S, S, S^{2},+\right)$, is determined by $a^{\varphi}$ and $b^{\varphi}$, which have to be idempotent again. Hence $E$ consists at most of the 3.3 mappings $\varphi_{1}, \ldots, \varphi_{9}$ listed in the following table, and clearly each of them is an endomorphism of $(S,+)$.

\begin{tabular}{l|c|c|c|c|c} 
& $a$ & $b$ & $a+b$ & $b+a$ & $z$ \\
\hline$\varphi_{1}\left(=\gamma_{a}\right)$ & $a$ & $a$ & $a$ & $a$ & $a$ \\
$\varphi_{2}(=\epsilon)$ & $a$ & $b$ & $a+b$ & $b+a$ & $z$ \\
$\varphi_{3}$ & $a$ & $z$ & $z$ & $z$ & $z$ \\
\hline$\varphi_{4}$ & $b$ & $a$ & $b+a$ & $a+b$ & $z$ \\
$\varphi_{5}\left(=\gamma_{b}\right)$ & $b$ & $b$ & $b$ & $b$ & $b$ \\
$\varphi_{6}$ & $b$ & $z$ & $z$ & $z$ & $z$ \\
\hline$\varphi_{7}$ & $z$ & $a$ & $z$ & $z$ & $z$ \\
$\varphi_{8}$ & $z$ & $b$ & $z$ & $z$ & $z$ \\
$\varphi_{9}\left(=\gamma_{2}\right)$ & $z$ & $z$ & $z$ & $z$ & $z$ \\
\hline$\varphi_{2}+\varphi_{2}$ & $a$ & $b$ & $z$ & $z$ & $z$ \\
$\varphi_{1}+\varphi_{5}$ & $a+b$ & $a+b$ & $a+b$ & $a+b$ & $a+b$
\end{tabular}

Both last rows show that $E$ is not closed with respect to addition, and for instance the element $\varphi_{2}+\varphi_{2}=\tau$ of the semi-near-ring $(\langle E\rangle,+, \cdot)^{4}$ is not

"We remark that the distributively generated semi-near-ring $(\langle E\rangle,+, \cdot)$ consists of 41 elements and does not contain an isomorphic image of the semiring $(S,+$,$) .$ 
distributive (not even with respect to elements contained in $E$ ) since

$$
\varphi_{1} \tau+\varphi_{5} \tau=\varphi_{1}+\varphi_{5}=\gamma_{a+b} \neq \varphi_{9}=\gamma_{a+b} \tau=\left(\varphi_{1}+\varphi_{5}\right) \tau \text {. }
$$

Hence $E$ is not strongly embeddable in a semiring. In order to prove that $E$ is weakly embeddable, let $(R,+, \cdot)$ be the semiring of all endomorphisms of the commutative semigroup $([S],+)$ which is the homomorphic image of $(S,+)$ defined by

$$
[a]=\{a\}, \quad[b]=\{b\}, \quad[a+b]=[b+a]=[z]=\{a+b, b+a, z\} .
$$

Obviously $R$ consists of the 9 endomorphisms $\psi_{i}=f\left(\varphi_{i}\right)$ given in the table above by identifying the last three columns, formally written as

$$
[x]^{\psi_{i}}=\left[x^{\Phi_{i}}\right], \text { for all } x \in S .
$$

Moreover, $f$ is the desired monomorphism and even from $E$ onto $R$. This is clear from our construction, but we give a formal proof of (3.1): If $\varphi_{i}+\varphi_{j}=\varphi_{k}$ is defined in $E$, we have by (3.3) for all $x \in S$

$$
[x]^{\psi_{k}}=\left[x^{\varphi_{k}}\right]=\left[x^{\varphi_{i}}+x^{\varphi}{ }_{j}\right]=\left[x_{i}^{\varphi}\right]+\left[x_{j}^{\varphi}\right]=[x]^{\psi_{i}}+[x]^{\psi_{j}} \text {. }
$$

Observe that in $(R,+, \cdot)$ we have $\psi_{2}+\psi_{2}=\psi_{2}$ and $\psi_{1}+\psi_{5}=\psi_{9}$.

Example 2. We obtain a semiring $(S,+, \cdot)$ on $S=\{a, b, o\}$ defining

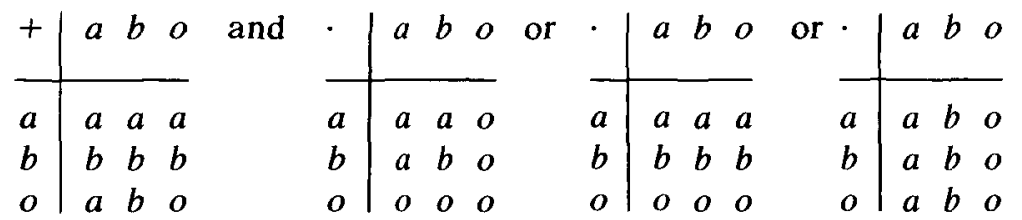

with each one of the given multiplications. The semigroups used are well known, and also most of the equations proving distributivity become trivial. Clearly $S^{2}=S$, and a noncommutative semigroup $(S,+)$ with zero is not subcommutative. Moreover, similar examples are obtained replacing $\{a, b\}$ by a set consisting of more elements.

One easily checks that the set $E=E(S)$ of all endomorphisms of $(S,+)$ has 7 elements and that $(\langle E\rangle,+, \cdot)$ is not distributive. But we do not need these concrete considerations because of the following

Proposition 2. Let $(S,+)$ be a semigroup with zero o, containing two idempotents $a, b$ such that $a+b$ and $b+a$ are also idempotent and distinct. Then the set $E=E(S)$ of all endomorphisms of $(S,+)$ is not even weakly embeddable in a semiring.

Proof. Suppose that $E$ is weakly embedded in a semiring $(R,+, \cdot)$ and let us identify the partial algebra $(E,+, \cdot)$ with its image in $R$. For each idempotent $w$ of $(S,+)$ the constant mapping $\gamma_{w}$ is contained in $E$, and $E$ 
has $\omega=\gamma_{o}$ as zero and the identity mapping $\epsilon$ as identity. Now we calculate in $(R,+, \cdot)$

$$
\begin{gathered}
\omega+\gamma_{a}+\gamma_{b}+\gamma_{b}=\left(\omega+\gamma_{a}\right)\left(\epsilon+\gamma_{b}\right)=\omega+\gamma_{b}+\gamma_{a}+\gamma_{b}, \\
\gamma_{b}+\gamma_{b}+\gamma_{a}+\omega=\left(\gamma_{a}+\omega\right)\left(\gamma_{b}+\epsilon\right)=\gamma_{b}+\gamma_{a}+\gamma_{b}+\omega,
\end{gathered}
$$

obtaining $\gamma_{a+b}=\gamma_{a}+\gamma_{b}=\gamma_{b}+\gamma_{a}=\gamma_{b+a}$ in $(E,+, \cdot)$, which contradicts $a+b \neq b+a$.

Corollary. Let $(S,+)$ be an idempotent semigroup with zero. Then the set $E=E(S)$ of all endomorphisms of $(S,+)$ is weakly as well as strongly embeddable in a semiring if and only if $(S,+)$ is commutative.

\section{REFERENCES}

(1) A. Fröhlich, Distributively generated nearrings I, Proc. London Math. Soc. (3) 8 (1958), 76-99.

(2) M. P. GRILLET, Examples of semirings of endomorphisms of semigroups, $J$. Austral. Math. Soc. 11 (1970), 345-349.

(3) H. E. Heatherly and J. J. Malone, Some nearring embeddings, Quart. J. Math. Oxford (2) 20 (1969), 81-85.

(4) H. E. Heatherly and J. J. Malone, Some nearring embeddings (II), Quart. J. Math. Oxford (2) 21 (1970), 445-448.

(5) H. HogEWIJS, Semi-Nearrings-Embedding, Med. Konink. Acad. Wetensch. Lett. Schone Kunst. België Kl. Wetensch. 32 (1970), 3-11.

(6) W. G. VAN HOORN and B. VAN ROOTSElaAR, Fundamental notions in the theory of seminearrings, Comp. Math. 18 (1967), 65-78.

(7) B. VAN ROOTSElAAR, Algebraische Kennzeichnung freier Wortarithmetiken, Comp. Math. 15 (1963), 156-168.

(8) H. J. WEINERT, Ringe mit nichtkommutativer Addition I, Jber. Deutsch. Math.-Verein. 77 (1975), 10-27.

Technical University Clausthal,

D 3392 Clausthal-Zellerfeld, Germany 